\title{
Swift follow-up observations of INTEGRAL sources of unknown nature
}

\author{
J. Rodriguez ${ }^{1}$, J. A. Tomsick ${ }^{2}$, and S. Chaty ${ }^{1}$ \\ 1 Laboratoire AIM, CEA/DSM - CNRS - Université Paris Diderot, DAPNIA/SAp, 91191 Gif-sur-Yvette, France \\ e-mail: jrodriguez@cea.fr \\ 2 Space Sciences Laboratory, 7 Gauss Way, University of California, Berkeley, CA 94720-7450, USA
}

Received 6 December 2007 / Accepted 16 February 2008

\section{ABSTRACT}

\begin{abstract}
Context. Since its launch in 2002, INTEGRAL has discovered many new hard X-ray sources. A lot of them still lack sufficient positional accuracy, for finding counterparts at other wavelengths. Their true nature is, therefore, still unknown.

Aims. The goal of this study is to give an accurate X-ray position for 12 of these sources so as to further identify their counterpart at optical, infrared, and radio wavelengths, and to unveil their true nature. We also make use of the X-ray spectral parameters to tentatively distinguish between the various possible types.

Methods. We made use of X-ray observations with the X-ray telescope on-board the Swift observatory to refine the X-ray position to $3-5^{\prime \prime}$ accuracy, and performed 0.1-10 keV spectral analysis. We then searched the online catalogues (e.g. NED, SIMBAD, 2MASS, 2MASX, and NVSS) to search for counterparts at other wavelengths.

Results. For all sources, we give a refined X-ray position, provide X-ray spectral parameters, identify infrared counterparts, and give magnitudes at optical and ultra violet wavelengths seen with UVOT when observations are available. We confirm the nature of six sources formerly suspected to be AGN (IGR J02343+3229, J13149+4422, J14579-4308, J16385-2057, J18559+1535, J19378-0617). Our analysis first leads us to suggest that IGR J09523-6231 and IGR J10147-6354 are AGN. While the former has recently been confirmed as a Seyfert 1.5 AGN, we suggest the latter is a Seyfert 2. All other sources may be Galactic sources, in which case their spectral shape may suggest that they are X-ray binaries. In one case (IGR J19308+0530), the Galactic nature is confirmed through the identification of an F8 star as the counterpart. We favour a distance to the source not greater than $1 \mathrm{kpc}$. The source is likely to be a neutron star XRB or a CV. We also report the discovery of six serendipitous sources of unknown nature.
\end{abstract}

Key words. astrometry - stars: binaries: close - galaxies: Seyfert - X-rays: binaries - X-rays: galaxies

\section{Introduction}

Since its launch on October 17, 2002, the INTErnational Gamma-Ray Astrophysical Laboratory (INTEGRAL, Winkler et al. 2003) has detected about 250 sources that had previously never been seen or serendipitously detected once, and not studied. This has been made possible mainly thanks to the high sensitivity, the wide field of view (FOV) and high imaging resolution of the IBIS Soft Gamma-Ray Imager (ISGRI, Lebrun et al. 2003 ) sensitive in the $20-300 \mathrm{keV}$ energy range. These sources are named with the acronym IGR JRA \pm Dec and we will hereafter refer to them as IGRs ${ }^{1}$. Understanding the nature of those sources has a great importance and implication for several astrophysical questions. The identification of sources allows us to perform statistics on families of sources and source population studies, while individual studies of new sources allows us to better understand the physics of the emission of high energy radiation. This, in turn, can help us answer questions regarding the evolution of stars, galaxies, and/or have cosmological implications while studying Galactic or extra-galactic sources such as AGN and other quasars.

In a recent paper, Bodaghee et al. (2007) collected all the known parameters (such as the absorption column density $N_{\mathrm{H}}$,

\footnotetext{
1 An up-to-date online catalogue of all IGRs can be found at http://isdc . unige.ch/\$ \$sim\$rodrigue/html/igrsources. html
}

or the pulse period for Galactic sources, the redshift for AGN, etc.) of all sources detected by INTEGRAL during the first four years of activity, and tried to understand the different families of sources by testing and searching correlations between those parameters. Their catalogue, however, contains a large number of sources whose high energy position is accurate just at the arcmin level, the best accuracy achievable with INTEGRAL/ISGRI. In most cases this level of accuracy is not sufficient to unveil the nature of the source through the identification of counterpart at other wavelengths. In some cases, a tentative identification is given, mainly when an AGN is found within the INTEGRAL/ISGRI error box, but this is far from being secure as other possible counterpart usually lie in the few arcmin ISGRI error box.

Since the discovery of the first source by INTEGRAL, secure identification has been possible only through follow-up observations with softer X-ray telescopes, either by refinement of the X-ray position and identification of the optical/infrared counterpart (e.g. Rodriguez et al. 2006; Tomsick et al. 2006; Chaty et al. 2008), or by discovery of X-ray pulsations in the case of pulsars (e.g. Lutovinov et al. 2005). Here, we report the results of several pointed observations made with the Swift observatory (Gehrels et al. 2004), on a total of 12 sources that lacked precise localisation at soft X-ray energies. We start by introducing the observations and method of data reduction, before giving the results on each source, i.e., refined position, identification of the 
Table 1. Log of the Swift observations analysed in this paper.

\begin{tabular}{lllll}
\hline \hline $\begin{array}{l}\text { Name } \\
\text { (IGR) }\end{array}$ & $\begin{array}{l}\text { Swift Sequence } \\
\text { Number }\end{array}$ & $\begin{array}{l}\text { Observation } \\
\text { Date }\end{array}$ & $\begin{array}{l}\text { Exp. }^{\star} \\
(\mathrm{s})\end{array}$ & $\begin{array}{l}\text { Offset }^{\dagger} \\
\left.{ }^{\prime}\right)\end{array}$ \\
\hline J02343+3229 & 00037105001 & $2007-07-03$ & 2481 & 5.4 \\
& 00037105002 & $2007-07-04$ & 4265 & 5.4 \\
& 00037105004 & $2007-07-07$ & 6402 & 5.1 \\
& 00037105006 & $2007-07-07$ & 6821 & 5.1 \\
J09523-6231 & 00030927001 & $2007-05-12$ & 1027 & 1.9 \\
& 00030927002 & $2007-06-08$ & 2123 & 1.0 \\
& 00030927003 & $2007-06-14$ & 1867 & 2.4 \\
J10147-6354 & 00037048001 & $2007-11-01$ & 4538 & 0.8 \\
J11187-5438 & 00037051001 & $2007-09-26$ & 6905 & 7.0 \\
& 00037051002 & $2007-09-30$ & 16062 & 2.9 \\
J13149+4422 & 00037093001 & $2007-06-03$ & 11493 & 7.8 \\
& 00037093002 & $2007-09-19$ & 1228 & 4.8 \\
& 00037093003 & $2007-09-20$ & 2449 & 7.1 \\
J14579-4308 & 00036621001 & $2007-09-21$ & 10725 & 8.0 \\
& 00036621002 & $2007-09-25$ & 6401 & 7.8 \\
& 00036621003 & $2007-09-25$ & 4552 & 7.4 \\
J16385-2057 & 00036649001 & $2007-10-07$ & 4554 & 6.1 \\
& 00036649002 & $2007-10-08$ & 4606 & 6.3 \\
J18490-0000 & 00035092001 & $2006-03-05$ & 8432 & 3.0 \\
& 00035092002 & $2006-03-09$ & 3828 & 2.0 \\
J18559+1535 & 00036651001 & $2007-07-20$ & 3848 & 2.5 \\
& 00036651002 & $2007-07-29$ & 1309 & 2.2 \\
& 00036651003 & $2007-10-03$ & 593 & 4.0 \\
J19308+0530 & 00036651004 & $2007-10-18$ & 5130 & 6.5 \\
& 00035357002 & $2006-04-13$ & 2120 & 1.3 \\
J19378-0617 & 00035357004 & $2006-05-19$ & 4477 & 1.2 \\
& 00036652001 & $2007-08-07$ & 408 & 7.2 \\
& 00036652003 & $2007-09-26$ & 5713 & 4.3 \\
& 00037065001 & $2007-06-09$ & 5901 & 0.9 \\
\hline
\end{tabular}

^ XRT exposure time.

$\dagger$ Offset between the direction of the Swift pointing and the best INTEGRAL position for the given source.

counterpart, and X-ray spectrum. We discuss and summarise the results in the last part of the paper.

\section{Observations and data reduction}

Among all the Swift pointed observations of IGRs, we first restricted our analysis to sources whose fine (less than $\sim 10^{\prime \prime}$ ) soft X-ray position was not published anywhere. We, then, disregarded the sources that were not detected in single pointings, because a non detection can have several reasons (absorption, under-exposures, variability, transience, etc.) that does not help in unraveling the type of a source. We also rejected the sources that were not observed in photon mode by the XRT, as no fine position can be obtained. In the remaining sample, we focused on sources for which either a secure identification was not given in Bodaghee et al. (2007) or those for which we found a mismatch between the X-ray position and the proposed association. Our analysis contains a sample of twelve sources. The observing $\log$ for these sources is reported in Table 1. All our results are first based on observations made with the XRT (Burrows et al. 2005) and UVOT (Roming et al. 2005) telescopes onboard the Swift observatory. The XRT is a focusing X-ray telescope with an effective area of $110 \mathrm{~cm}^{2}$, and a FOV of about 23'. It has an imaging resolution of $18^{\prime \prime}$ between 0.1 and $10 \mathrm{keV}$, and has a location accuracy for point source as low as $\sim 3^{\prime \prime}$.

The XRT data were reduced within the HEASOFT package v. 6.3.2. We produced level 2 data with the xrtpipeline v0.11.5 that processes the raw data to obtain clean data products, i.e. images, spectra and light curves used for the scientific analysis. For each pointing, we estimated the X-ray position of the X-ray sources with the task xrtcentroid v.0.2.7. In the various cases where several pointings were available for the same source the final position is the mean of all positions obtained from the individuals pointings. As the error computed by this task includes various effects (especially some systematic effects and satellite misalignment), the error we report here is also the average of all individual errors.

Spectra and light curves were extracted with xselect v2.4. The source spectra and light curves were obtained from a circle of 20 pixels $\left(\sim 47^{\prime \prime}\right)$ radius centred on the best source position. This ensures that $90 \%$ of the PSF is enclosed in this region. Background spectra and light curves were extracted from a region of the detector free of sources and of 40 pixels radius. Exposure maps were produced with xrtexpomap and summed within XIMAGE. The ancillary response files were generated with the tool xrtmkarf $v 0.5 .5$, and corrected with the exposure maps at the position of the source. The resultant spectra were rebined so as to at least have 20 counts per channel allowing the chistatistics to be used in xspec v11.3.2ag. If this criterion was not achievable, the Cash-statistic was used instead. In the cases where several pointings are available the spectra were averaged together unless large variability was seen in the light curve. The spectra were fitted between $0.5 \mathrm{keV}$ and $\sim 8 \mathrm{keV}$ depending on the quality of the high energy bins.

The UVOT is an UV/optical telescope, whose design is based on the OM onboard the XMM-Newton observatory. It has a $17^{\prime} \times 17^{\prime}$ FOV with an angular resolution of about $2^{\prime \prime}$ depending of the filter used. It can observe a given field through several filters, or grisms to perform spectroscopy. The UVOT data (when available) discussed in this paper were obtained through one or more of the following filters: $V$ (5000-6000 $\AA$ ),

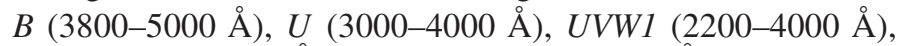

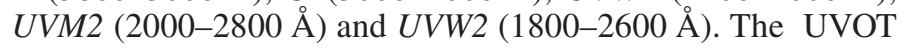
individual exposures of a single observation were summed with uvotimsum while magnitudes and upper limits were estimated at the best X-ray position obtained with XRT with uvotsource as explained in the UVOT analysis thread ${ }^{2}$ by comparison with a region free of sources taken as background reference. Note that, as indicated in the UVOT analysis threads, regions of, respectively, $6^{\prime \prime}$ radius for the $U, B$, and $V$ filters and $12^{\prime \prime}$ radius, for the $U V W 1, U V W 2, U V M 2$ filters, where given as an input to uvotsource for the computation of the magnitudes. The typical errors are $0.2 \mathrm{mag}$.

\section{Results}

The main results, X-ray position, presence of an infrared counterpart and its type are reported in Table 2, the IR, optical magnitudes found in the literature, and the magnitudes obtained from the analysis of the UVOT data when available are reported in Table 3. The spectral results are reported in Table 4. All errors are given at the $90 \%$ confidence level. The luminosities reported in Table 4 are estimated at a distance of $1 \mathrm{kpc}$ for sources of unknown type. For the confirmed AGNs, we used the redshift reported in Table 2 and $H_{0}=65 \mathrm{~km} \mathrm{~s}^{-1} \mathrm{Mpc}^{-1}$ to estimate it. Note that for all sources, the Swift/XRT position falls well within the $90 \%$ confidence INTEGRAL error box. In all cases there is a single source within the INTEGRAL error box, and except where further discussed below, there are no other bright sources in the

\footnotetext{
2 http://swiftsc.gsfc.nasa.gov/docs/swift/analysis/ threads/uvot_threads.html
} 
Table 2. X-ray position (equatorial and Galactic), presence of an IR counterpart and reddshift of the 12 sources studied with Swift/XRT.

\begin{tabular}{|c|c|c|c|c|c|c|c|c|}
\hline $\begin{array}{l}\text { Name } \\
\text { (IGR) }\end{array}$ & $\begin{array}{l}\text { RA } \\
(\mathrm{J} 2000)\end{array}$ & $\begin{array}{l}\text { Dec } \\
(\mathrm{J} 2000)\end{array}$ & $\begin{array}{c}\text { Error } \\
(")\end{array}$ & $\begin{array}{l}l \\
\left({ }^{\circ}\right)\end{array}$ & $\begin{array}{l}b \\
\left({ }^{\circ}\right)\end{array}$ & $\begin{array}{c}\text { Counterpart } \\
\text { (infrared) }\end{array}$ & Type & Comment \\
\hline $\mathrm{J} 02343+3229$ & $02^{\mathrm{h}} 34^{\mathrm{m}} 19.9^{\mathrm{s}}$ & $+32^{\circ} 30^{\prime} 20^{\prime \prime}$ & 3.8 & 146.865 & -25.540 & yes/extended & Sey 2 & $z=0.016$ \\
\hline J09523-6231 & $09^{\mathrm{h}} 52^{\mathrm{m}} 20.5^{\mathrm{s}}$ & $-62^{\circ} 32^{\prime} 37^{\prime \prime}$ & 4.4 & 283.854 & -6.507 & yes (Fig. 1) & Sey 1.5 & $z=0.252^{\star}$ \\
\hline $\mathrm{J} 10147-6354$ & $10^{\mathrm{h}} 14^{\mathrm{m}} 15.2^{\mathrm{s}}$ & $-63^{\circ} 51^{\prime} 50^{\prime \prime}$ & 4.2 & 286.638 & -6.083 & yes/point & Sey $2(?)$ & \\
\hline J11187-5438 & $11^{\mathrm{h}} 18^{\mathrm{m}} 21.1^{\mathrm{s}}$ & $-54^{\circ} 37^{\prime} 32^{\prime \prime}$ & 3.7 & 289.640 & +5.826 & yes/point & ? & \\
\hline $\mathrm{J} 13149+4422$ & $13^{\mathrm{h}} 15^{\mathrm{m}} 17.3^{\mathrm{s}}$ & $+44^{\circ} 24^{\prime} 26^{\prime \prime}$ & 3.8 & 108.983 & +72.069 & yes/extended & Sey 2 & $z=0.035$ \\
\hline $\mathrm{J} 14579-4308$ & $14^{\mathrm{h}} 57^{\mathrm{m}} 41.3$ & $-43^{\circ} 07^{\prime} 57^{\prime \prime}$ & 3.8 & 326.114 & +13.984 & yes/extended & Sey 2 & $z=0.016$ \\
\hline J16385-2057 & $16^{\mathrm{h}} 38^{\mathrm{m}} 31.1^{\mathrm{s}}$ & $-20^{\circ} 55^{\prime} 25^{\prime \prime}$ & 3.6 & 357.728 & +17.020 & yes/extended & Sey 1 & $z=0.027$ \\
\hline $\mathrm{J} 18490-0000$ & $18^{\mathrm{h}} 49^{\mathrm{m}} 01.6^{\mathrm{s}}$ & $-0^{\circ} 01^{\prime} 18^{\prime \prime}$ & 3.7 & 32.646 & +0.510 & faint IR/point & XRB (?) & \\
\hline $\mathrm{J} 18559+1535$ & $18^{\mathrm{h}} 56^{\mathrm{m}} 00.6^{\mathrm{s}}$ & $+15^{\circ} 37^{\prime} 58^{\prime \prime}$ & 3.6 & 47.409 & +6.072 & yes/point & Sey 1 & $z=0.084$ \\
\hline $\mathrm{J} 19308+0530$ & $19^{\mathrm{h}} 30^{\mathrm{m}} 50.9^{\mathrm{s}}$ & $+05^{\circ} 30^{\prime} 57^{\prime \prime}$ & 4.3 & 42.381 & -6.186 & yes/point & neutron star XRB or $\mathrm{CV}$ & F8 star \\
\hline J19378-0617 & $19^{\mathrm{h}} 37^{\mathrm{m}} 33.1^{\mathrm{s}}$ & $-06^{\circ} 13^{\prime} 04^{\prime \prime}$ & 3.5 & 32.591 & -13.074 & yes/extended & Sey 1.5 & $z=0.011$ \\
\hline $\mathrm{J} 23524+5842$ & $23^{\mathrm{h}} 52^{\mathrm{m}} 22.0^{\mathrm{s}}$ & $+58^{\circ} 45^{\prime} 31^{\prime \prime}$ & 4.5 & 115.323 & -3.238 & yes/point & ? & \\
\hline
\end{tabular}

^ From Masetti et al. (2008).

Table 3. IR, Optical and UV apparent magnitudes obtained from the literature and online catalogues, and, when available, from the analysis of the UVOT data. Upper limits are given at the 5- $\sigma$ level.

\begin{tabular}{llll}
\hline \hline $\begin{array}{l}\text { Source } \\
\text { IGR) }\end{array}$ & IR & \multicolumn{1}{c}{ Magnitudes } & \\
\hline Op2343+3229 & $J=10.021, H=9.184, K=8.772$ & $B=13.7$ & $\mathrm{UV}$ \\
$\mathrm{J} 09523-6231$ & & & $U=17.7, U V W 2=18.3$ \\
$\mathrm{~J} 10147-6354$ & $J=14.831, H=13.874, K=12.53$ & & $U=18.6, U V M 2>19.3, U V W 1>19.0$ \\
$\mathrm{~J} 11187-5438$ & $J=15.534, H=14.869, K=14.455$ & & \\
$\mathrm{~J} 13149+4422$ & $J=12.248, H=11.624, K=10.821$ & $B=16.5$ & $U V M 2=16.9, U V W 1=16.7$ \\
$\mathrm{~J} 14579-4308$ & $J=10.745, H=9.918, K=9.578$ & $B=15$ & \\
$\mathrm{~J} 16385-2057$ & $J=12.527, H=11.644, K=11.106$ & & \\
$\mathrm{~J} 18490-0000$ & $K=14.159$ & $V>20.2$ & \\
$\mathrm{~J} 18559+1535$ & $J=13.639, H=12.584, K=11.438$ & & $U V M 2>19.4, \mathrm{UVW} 2>20.2$ \\
$\mathrm{~J} 19308+0530$ & $J=9.617, H=9.245, K=9.130$ & $V=11.0, B=11.3$ & $U<11.9, U V M 2=12.5, U V W 2=13.7$ \\
$\mathrm{~J} 19378-0617$ & $J=10.88, H=10.141, K=9.666$ & $V=15.35, B=16.12$ & $U V W 1=14.4$ \\
$\mathrm{~J} 23254+5842$ & $J=16.127, H=15.059, K=13.9155$ & & $U>20.7$ \\
\hline
\end{tabular}

FOV, although there may be some slight excesses in some of the fields. The infrared (IR) counterparts are either found in the 2MASS point source catalogue, or the 2MASX extended source catalogue. Below, we discuss some individual properties for each source.

\subsection{IGR J02343+3229}

IGR J02343+3229 was discovered with INTEGRAL by Burenin et al. (2006) and Krivonos et al. (2007), and promptly associated with NGC 973 a likely Sey 2 AGN (Burenin et al. 2006). The average XRT position is 3.7" away from the reported position for NGC 973. The angular size of the galaxy is 3.98', which renders the association of the X-ray source and the Galaxy very likely. We found a possible counterpart in the 2MASX catalogue of extended sources. 2MASX J02342010+3230200 is 2.0" away from the centre of the Swift/XRT error box. Both objects fall well within the XRT error, further increasing the probability that IGR J02343+3229 is an AGN. This AGN is at $z=0.016$ as reported in NED from various sources. We examined the UVOT data of the third exposure that contains coverage in the $U$ and UVW2 filters. The best XRT position of IGR J02343+3229 clearly contains the nucleus of a galaxy detected in both filters. The magnitudes obtained at the best XRT position within the recommended 6 "and $12^{\prime \prime}$ radius regions of extraction are reported in Table 3.

We remark the presence of an additional source and another possible faint excess in the field. Both features are outside
Table 4. Spectral parameters of the 12 sources studied with Swift/XRT.

\begin{tabular}{lllll}
\hline \hline $\begin{array}{l}\text { Name } \\
(\text { IGR })\end{array}$ & $\begin{array}{l}N_{\mathrm{H}} \\
\times 10^{22} \mathrm{~cm}^{-2}\end{array}$ & $\Gamma$ & Flux $^{\star}$ & Lumin. $^{\dagger}$ \\
\hline $\mathrm{J} 02343+3229$ & $2.2 \pm 0.4$ & $1.3 \pm 0.2$ & $1.0 \times 10^{-11}$ & $6.7 \times 10^{42}$ \\
$\mathrm{~J} 09523-6231$ & $8_{-4}^{+5}$ & $2.3_{-1.1}^{+1.3}$ & $9.1 \times 10^{-12}$ & $1.5 \times 10^{43}$ \\
$\mathrm{~J} 10147-6354$ & $2.0_{-1.1}^{+1.6}$ & $1.7_{-0.8}^{+0.9}$ & $2.1 \times 10^{-12}$ & $2.5 \times 10^{32}$ \\
$\mathrm{~J} 11187-5438$ & $0.28_{-0.07}^{+0.08}$ & $1.5 \pm 0.14$ & $3.4 \times 10^{-12}$ & $4.1 \times 10^{32}$ \\
$\mathrm{~J} 13149+4422$ & $5.2_{-1.0}^{+1.5}$ & $1.7_{-0.3}^{+0.4}$ & $1.2 \times 10^{-11}$ & $4.1 \times 10^{43}$ \\
$\mathrm{~J} 14579-4308$ & $20 \pm 4$ & $2.9 \pm 0.6$ & $1.8 \times 10^{-11}$ & $1.2 \times 10^{43}$ \\
$\mathrm{~J} 16385-2057$ & $0.21 \pm 0.04$ & $2.1 \pm 0.1$ & $7.7 \times 10^{-12}$ & $1.4 \times 10^{43}$ \\
$\mathrm{~J} 18490-0000$ & $5 \pm 2$ & $1.8_{-0.6}^{+0.7}$ & $6.4 \times 10^{-12}$ & $7.6 \times 10^{32}$ \\
$\mathrm{~J} 18559+1535$ & $0.7 \pm 0.1$ & $1.6 \pm 0.1$ & $1.4 \times 10^{-11}$ & $2.5 \times 10^{44}$ \\
$\mathrm{~J} 19308+0530$ & $<0.3^{\ddagger}$ & $3.0_{-0.5}^{+1.4}$ & $8.2 \times 10^{-14}$ & $9.8 \times 10^{30}$ \\
$\mathrm{~J} 19378-0617$ & $0.15 \pm 0.05$ & $2.5 \pm 0.2$ & $4.4 \times 10^{-11}$ & $1.3 \times 10^{43}$ \\
$\mathrm{~J} 23524+5842$ & $6_{-2}^{+4}$ & 2 frozen & $2.9 \times 10^{-12}$ & $3.4 \times 10^{32}$ \\
\hline
\end{tabular}

* 2-10 keV unabsorbed $\left(\mathrm{erg} \mathrm{cm}^{-2} \mathrm{~s}^{-1}\right)$.

2-10 keV unabsorbed luminosity $\left(\mathrm{erg} \mathrm{s}^{-1}\right.$ ).

$90 \%$ upper limit.

the INTEGRAL error box. The position of the source (named SWIFT J023405.1+322707) is reported in Table 5. It lies 2.5" from HD 15896 (=2MASS J02340529+3227074) a K0 star, with 
Table 5. Best XRT positions of serendipitous sources found in this study.

\begin{tabular}{llll}
\hline \hline $\begin{array}{l}\text { Name } \\
\text { (SWIFT) }\end{array}$ & RA $_{\mathrm{J} 2000}$ & Dec $_{\mathrm{J} 2000}$ & $\begin{array}{l}\text { Error } \\
\left({ }^{\prime \prime}\right)\end{array}$ \\
\hline $\mathrm{J} 023405.1+322707$ & $02^{\mathrm{h}} 34^{\mathrm{m}} 05.1^{\mathrm{s}}$ & $+32^{\circ} 27^{\prime} 07^{\prime \prime}$ & $4.1^{\prime \prime}$ \\
$\mathrm{J} 095238.4-622316$ & $09^{\mathrm{h}} 52^{\mathrm{m}} 38.4^{\mathrm{s}}$ & $-62^{\circ} 23^{\prime} 16^{\prime \prime}$ & $4.1^{\prime \prime}$ \\
$\mathrm{J} 145704.4-430020$ & $14^{\mathrm{h}} 57^{\mathrm{m}} 04.4^{\mathrm{s}}$ & $-43^{\circ} 00^{\prime} 20^{\prime \prime}$ & $3.8^{\prime \prime}$ \\
$\mathrm{J} 145729.9-430231$ & $14^{\mathrm{h}} 57^{\mathrm{m}} 29.9^{\mathrm{s}}$ & $-43^{\circ} 02^{\prime} 31^{\prime \prime}$ & $4.0^{\prime \prime}$ \\
$\mathrm{J} 145746.9-430056$ & $14^{\mathrm{h}} 57^{\mathrm{m}} 46.9^{\mathrm{s}}$ & $-43^{\circ} 00^{\prime} 56^{\prime \prime}$ & $4.2^{\prime \prime}$ \\
$\mathrm{J} 145702.3-430128$ & $14^{\mathrm{h}} 57^{\mathrm{m}} 02.3^{\mathrm{s}}$ & $-43^{\circ} 01^{\prime} 28^{\prime \prime}$ & $4.0^{\prime \prime}$ \\
\hline
\end{tabular}
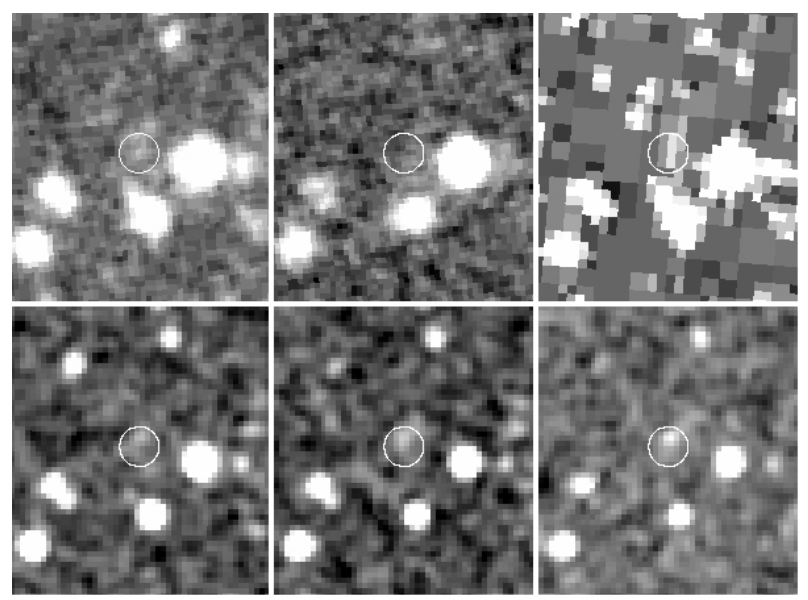

Fig. 1. 46" $\times 59^{\prime \prime}$ finding charts of the field around IGR J09523-6231. From left to right and top to bottom DSS II infrared, red, blue images, and 2MASS $J, H$ and $K$-band images. In all images, the small circle represents the $S$ wift/XRT error box.

$B=8.74, V=7.65, J=5.754, H=5.259, K=5.087$, which may suggest the source has a Galactic origin.

We combined the four observations to perform a spectral analysis of IGR J02343+3229. The average spectrum has $1437 \mathrm{cts}$ for a total of $19910 \mathrm{~s}$ exposure. An absorbed power law fits the data well with a reduced $\chi^{2}$ (hereafter $\chi_{v}^{2}$ ) of 1.11 for 63 degrees of freedom (d.o.f.). The parameter values are reported in Table 2. The value of $N_{\mathrm{H}}$ is about 40 times higher than the mean absorption along the line of sight, which indicates that IGR J02343+3229 is an intrinsically absorbed AGN. Note that intrinsic absorption is expected in the case of a Sey 2 AGN.

\subsection{IGR J09523-6231}

IGR J09523-6231 was first reported in the third edition of the IBIS catalogue (Bird et al. 2007). The source was only detected in the $20-40 \mathrm{keV}$ energy range at a low significance of $5.3 \sigma$ over $290 \mathrm{ks}$ of observation (Bird et al. 2007). Only one X-ray source is found in the INTEGRAL error box. It is, however, extremely weak and the positions obtained from the three Swift/XRT pointings can differ by few arcsec (up to 4). There is nothing reported in the various online catalogue at less than $10^{\prime \prime}$ from the average $\mathrm{X}$-ray position. Figure 1 shows the DSS II R-band and infrared, and the 2MASS $J, H$, and $K$-band images. As can be seen in Fig. 1, a weak source is found within the Swift error box in the 2MASS and DSS II images. The source may even be blended or extended as seen from the infrared images. We examined the UVOT data for the presence of a possible counterpart at the position of the source. A faint source is visible in the $U$-band only (Table 3).
We remark the presence of another X-ray source in the Swift/XRT FOV. It is well outside the INTEGRAL error box. Its position is reported in Table 5. This additional source (named SWIFT J095238.4-622316) is within the 14" ROSAT error box of 1RXS J095237.2-622310, which suggests the two sources are the same. A counterpart with $m_{U V M 2}=17.2 \pm 0.2$ is found in the XRT error box of SWIFT J095238.4-622316. This source is unfortunately outside the UVOT FOV of the pointings made with the $U$ and $U V W 1$ filters.

We combined the three observations to perform a spectral analysis. The spectrum has $215 \mathrm{cts}$ for a total of $5017 \mathrm{~s}$ exposure. An absorbed power law fits the data well $\left(\chi_{v}^{2}=0.9\right.$ for 6 d.o.f. $)$. The fitted value of $N_{\mathrm{H}}$ is about 40 times higher than the average value of the absorption along the line of sight, which indicates that IGR J09523-6231 is intrinsically absorbed. The detection of the source in the $U$-filter and the high X-ray absorption first led us to think the object was a Sey 2 AGN, since in those objects, the optical emission is thought to be produced outside of the absorbing matter affecting the X-ray emission. This despite the position of the source close to the Galactic plane (Table 2) which may have rather pointed towards a Galactic source, as AGN tend to statistically be found at high Galactic latitudes. In a very recent work, Masetti et al. (2008), showed through optical spectroscopy, that IGR J09523-6231 was indeed an AGN. They refined the type to a Sey 1.5 .

\subsection{IGR J10147-6354}

IGR J10147-6354 was first reported by Bird et al. (2007). The source was detected in the 40-100 keV energy range at a significance of $4.9 \sigma$ during $1340 \mathrm{ks}$ of observation. The best XRT position is well within the ISGRI error box of $5^{\prime}$.

There is a single source in the 2MASS point source catalogue within $5^{\prime \prime}$ from the XRT position. 2MASS J10141554-6351500 is at $2.1^{\prime \prime}$ away from the centre of the Swift error box. It is also well detected in the UVOT $U V W 2$ filter.

The XRT spectrum has $93 \mathrm{cts}$ for a total exposure of $4538 \mathrm{~s}$. An absorbed power law fits the data well, with a C-statistic value $=32.6$ for 29 bins. The value of $N_{\mathrm{H}}$ is a factor about 7.3 times higher than the average value of the absorption along the line of sight which indicates that the absorption is mostly intrinsic to the source. The detection of a bright UV counterpart, as for the former source, suggests either a very close Galactic or a far and bright extragalactic object. Again, the low Galactic latitude would rather tend to point towards a Galactic source rather than an extragalactic one. Dereddening the magnitudes with the absorption on the line of sight does, however, not lead to any known stellar spectral type for a Galactic source. This, the detection in the UV-band, and the intrinsic absorption of the source would tend to favour a Sey 2 AGN.

\subsection{IGR J11187-5438}

IGR J11187-5438 was first reported by Bird et al. (2007). The source was detected at a $18-60 \mathrm{keV}$ significance of $6.3 \sigma$ during $1016 \mathrm{ks}$ of observations. There is a single source in the 2MASS point source catalogue within $5^{\prime \prime}$ from the XRT position. 2MASS J11182121-5437286 is at 3.5" away from the centre of the Swift error box. The source is also visible in the DSS II IR and $R$-Band images, although it is quite weak in the latter. The UVOT was not operating during either of the observations. 
We combined the two observations to perform a spectral analysis. The average spectrum has 1328 cts for a total of 21888 s exposure. An absorbed power law fits the data well $\left(\chi_{v}^{2}=0.91\right.$ for 55 d.o.f.). The value of the absorption is compatible with the Galactic absorption along the line of sight, which may mean that the source is not intrinsically absorbed.

\subsection{IGR J13149+4422}

IGR J13149+4422 was first reported in Sazonov et al. (2007) and Krivonos et al. (2007). The latter give an IBIS position of $\mathrm{RA}_{\mathrm{J} 2000}=13^{\mathrm{h}} 14^{\mathrm{m}} 58^{\mathrm{s}}$ Dec $_{\mathrm{J} 2000}=+44^{\circ} 23^{\prime}$ with a $1-\sigma$ uncertainty as high as $2.1^{\prime}$. It was then tentatively identified with Mrk 248 a Sey 2 AGN at $z=0.037$ (Sazonov et al. 2007). The best Swift/XRT position is $3.56^{\prime}$ from the IBIS position, therefore within the $90 \%$ confidence error box. It is compatible with the best reported position of UGC 8327 NED02 (at $\mathrm{RA}_{\mathrm{J} 2000}=13^{\mathrm{h}} 15^{\mathrm{m}} 17.270^{\mathrm{s}}, \operatorname{Dec}_{\mathrm{J} 2000}=+44^{\circ} 24^{\prime} 25^{\prime \prime} .60$ according to the latest measurements available in NED, thus at $0.13^{\prime \prime}$ from the centre of the Swift error box). Note that this is also at $16^{\prime \prime} 8$ from the position reported in Bodaghee et al. (2007), and in SIMBAD for Mrk 248. We remark that SIMBAD reports an earlier measurement than NED, and also mentions Mrk 248 as being UGC 8327, a pair of interacting galaxies (UGC 8327). NED, on the other hand, associates Mrk 248 with one of the components of the pair of interacting galaxies (UGC 8327 NED02). Mrk 248 is also reported in the 2MASS extended source catalogue as 2MASX J13151725+4424259 whose best position is at only $0.64^{\prime \prime}$ away from the best X-ray position. The 2MASS source is also classified as an emission line galaxy in SIMBAD. An extended or blended source is also clearly visible in the UVOT UVM2 and UVW1 images within the XRT error box. The spatial coincidence between all sources likely indicates that IGR J13149+4422 is a Sey 2 AGN.

We extracted a spectrum from three pointings. The spectrum has $964 \mathrm{cts}$ for a total of $15116 \mathrm{~s}$. It is well fitted by an absorbed power law $\left(\chi^{2}=1.11\right.$ for 32 d.o.f.). We note the presence of positive residuals at low energy. The value of $N_{\mathrm{H}}$ is about 430 times higher than the average absorption along the line of sight, which indicates that the source is an intrinsically absorbed AGN as would be expected in the case of a Sey 2 AGN.

\subsection{IGR J14579-4308}

IGR J14579-4308 was first reported by Kalemci et al. (2005) from an IBIS/ISGRI observation of SN 1006. It was promptly suggested to be an AGN due to its positional coincidence with VV 780 a Sey 2 AGN. Revnivtsev et al. (2006) later reported the presence of the source from a survey of the Galactic Crux arm tangent. The XRT images show that the field is crowded with about five bright sources in the field of view (Fig. 2). Only the brightest source is, however, found within the maximum of $6^{\prime}$ error box of ISGRI (Revnivtsev et al. 2006). It is labeled 1 in Fig. 2. The best XRT position is $42^{\prime \prime}$ away from the reported position of IC 4518/ VV 780 which rules out an association between the X-ray source and the AGN. Bird et al. (2007) suggested a possible association of the INTEGRAL source with IC 4518A, a Sey 2 galaxy as inferred from optical spectroscopy (Masetti et al. 2007), also the western component of a pair of interacting galaxies named MCG-07-31-001. The best Swift position is, indeed, just 1.48" away from the position of IC 4518A (also named VV 780 NED01) as reported in NED. Our Swift analysis, therefore, strengthens the association of both objects,

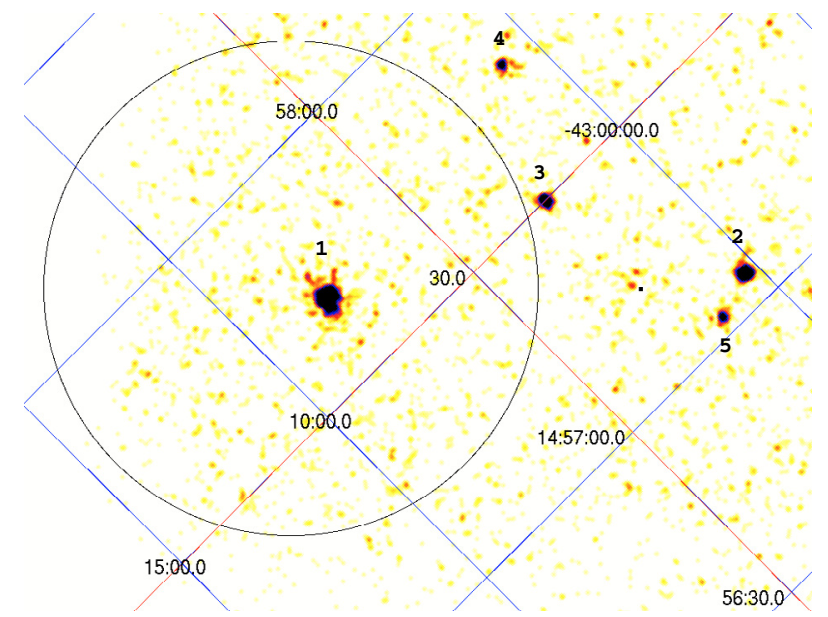

Fig. 2. Swift XRT image of the field around IGR J14579-4308. The 5 sources are indicated with numbers. Source 1 is the X-ray counterpart to IGR J14579-4308. The big circle is the maximum IBIS error box of 6 ' reported in Revnivtsev et al. (2006).

and we confirm that IGR J14579-4308 is a Sey 2 galaxy. Note that, as for IGR J13149+4422, the position of IGR J14579-4308 reported in Bodaghee et al. (2007) and in SIMBAD is that of the pair of interacting galaxies, while we identify here one of the components of this double system as the best counterpart to the INTEGRAL source thanks to the refined position. The best positions for the other sources are reported in Table 5. Only source 2 in Fig. 2 has an infared counterpart reported in 2MASS. 2MASS J14570433-4300187 indeed lies 1'.5 from SWIFT J145704.4-430020. No UVOT data are available from any of the observing sequences.

We combined the three observations to perform a spectral analysis. The average spectrum of IGR J14579-4308 has 703 cts for a total of $21685 \mathrm{~s}$ exposure. A simple absorbed power law does not fit the data well $\left(\chi_{v}^{2}=2.88\right.$ for 29 d.o.f. $)$. A large excess is visible below $2 \mathrm{keV}$. Adding a black body to the model improves the fit to $\chi_{v}^{2}=1.25$ for 27 d.o.f. The black body radiation is not absorbed and has a temperature of $0.28_{-0.06}^{+0.11} \mathrm{keV}$. The best parameters of the other spectral components (power law and $N_{\mathrm{H}}$ ) are reported in Table 2 . Replacing the black body by a disc model (diskpn in XSPEC) provides a good description of the spectrum $\left(\chi_{v}^{2}=1.28\right.$ for 27 d.o.f. $)$. The inner disc temperature is $0.5_{-0.2}^{+0.7} \mathrm{keV}$. The high value of the absorption indicates that the object is intrinsically highly absorbed, as would be expected in a Sey 2 AGN. Further spectral results will be reported in Kalemci et al. (in prep.).

\subsection{IGR J16385-2057}

IGR J16385-2057 was first reported by Bird et al. (2007). Based on a positional coincidence with 1RXS J163830.9-205520 and Oph J163830-2055, it was suggested to be the X-ray counterpart to those objects. Optical spectra allowed Masetti et al. (2006a) to tentatively classify it as a Sey 1 at $z=$ 0.027. The Swift/XRT position is 2.5" away from the position of 2MASX J16383091-2055246 an AGN at $z=0.026$ (Hasegawa et al. 2000). This object is also compatible with Oph J163830-2055, and therefore the tentative identification as a Sey 1 seems confirmed by the refined XRT position. Note that the Swift position is also 5.73" from 1RXS J163830.9-205520, well within the ROSAT error box (7"). No UVOT data are available for this source. 
We combined the two observations to perform a spectral analysis. The average spectrum of IGR J16385-2057 has 2195 cts for a total of $9168 \mathrm{~s}$ exposure. An absorbed power law fits the data well $\left(\chi_{v}^{2}=1.27\right.$ for 85 d.o.f.). The value of the absorption is a factor of two higher than the total absorption along the line of sight. This may indicate that a part of the absorbing material is intrinsic to the object.

\subsection{IGR J18490-0000}

IGR J18490-0000 was first reported by Molkov et al. (2004) from a survey of the Sagittarius Arm tangent region. Nothing more is known about this source. There is a single 2MASS point source within the Swift/XRT error box. 2MASS J184901820001190 lies $3.55^{\prime \prime}$ from the centre of the Swift error box. It has a well-measured magnitude only in the $K$-band, while it is not detected in the UVOT $V$-filter.

We extracted an average spectrum from the two pointings. The spectrum has 441 counts for a total of $12208 \mathrm{~s}$ and is well fitted by an absorbed power law ( $\chi_{v}^{2}=0.40$ for 17 d.o.f.). The value of $N_{\mathrm{H}}$ is about 3 times higher than the average value of the absorption along the line of sight. This may indicate that IGR J18490-0000 is intrinsically absorbed. This source is likely a Galactic X-ray binary because of the presence of a point source $K$-band counterpart, its spectrum intrinsically absorbed and typical of an XRB, and its position towards the Sagittarius Arm.

\subsection{IGR J18559+1535}

The first mention of an INTEGRAL detection of this source is reported in Bird et al. (2006), as 2E 1853.7+1534, a Sey 1 AGN. The Swift/XRT position is about 12" away from the best reported position for 2E 1853.7+1534/2MASX J18560128+1538059. There are no extended sources or NED objects within $10^{\prime \prime}$ of the best position. In the XRT error box there are, however, a $2 \mathrm{MASS}$ point source, 2MASS J18560056+1537584 at 0.7" and a ROSAT source 1RXH J185600.4+153757 at 2.89". The positional coincidence of these two sources probably indicates that they are related. We do not detect any source in the UVOT UVM2 and UVW2-filters at the best XRT positions. Masetti et al. (2006a) and Bikmaev et al. (2006) performed optical follow-up observations of the field of this object. Masetti et al. (2006a) suggested an association of this gamma-ray source with the ROSAT source, which allowed them to perform optical spectroscopy of the object. They identified it as a Sey 1 galaxy at $z=0.084 \pm 0.001$. Bikmaev et al. (2006) obtained an optical position exactly coincident with the Swift position of IGR J18559+1535. They also refine the redshift to $z=0.0844 \pm 0.0002$. Our X-ray refined position strongly confirms the identification of the high energy source as a Sey 1 galaxy.

We extracted an average spectrum from the four pointings. The spectrum has $1572 \mathrm{cts}$ for a total exposure of $8378 \mathrm{~s}$. The spectrum is well fitted with an absorbed power law $\left(\chi_{v}^{2}=0.90\right.$ for 68 d.o.f.). The value of $N_{\mathrm{H}}$ is about twice the value of the absorption along the line of sight which could indicate that the object has some intrinsic absorption, although at a low level, as would be expected from a Sey 1 AGN.

\subsection{IGR J19308+0530}

IGR J19308-0530 was first reported by Bird et al. (2006). It was detected at a $20-60 \mathrm{keV}$ significance of $6.6 \sigma$ for a total of 949 ks of observing time. Within the Swift/XRT error box lies a known star, TYC 486-295-1 also reported in the 2MASS catalogue as 2MASS J19305075+0530582. The average Swift/XRT position is just $2.5^{\prime \prime}$ away from the best position of TYC 486295-1 = 2MASS J19305075+0530582 a F8 star. This positional coincidence may suggest IGR J19308+0530 is a Low Mass/Intermediate Mass X-ray Binary. However, all DSII and 2MASS images are saturated at the position of TYC 486-2951/2MASS J19305075+0530582, and we do not exclude that this is actually a blend of sources. The source saturates the UVOT images in the $U, B$ and $V$-filters, and is clearly detected in the other filters (Table 3).

We extracted an average spectrum from the two pointings. The source is quite weak, with a 82 cts spectrum for a total exposure of $6556 \mathrm{~s}$. The spectrum is well fitted by an absorbed power law (C-statistic value 20.7 for 22 bins). The value of $N_{\mathrm{H}}$ is compatible with the value of the absorption along the line of sight. This indicates that the object is not intrinsically absorbed. As can be seen in Table 2 the power law photon index is quite soft. As such a steep power law may be indicative of a thermal spectrum, we replaced it by an absorbed absorbed black body. The fit is rather good (C-statistic value 26 for 23 bins). The best parameters are $N_{\mathrm{H}}<0.15 \times 10^{22} \mathrm{~cm}^{-2}(90 \%$ upper limit) and $k T=0.26 \pm 0.5 \mathrm{keV}$. Note that a bremsstrahlung also fits the spectrum well.

We dereddened the data with the value of $N_{\mathrm{H}}$ obtained from the spectral fit (Table 2) and the value of Galactic value of the absorption along the line of sight $\left(\sim 0.2 \times 10^{22} \mathrm{~cm}^{-2}\right)$. We then estimated the distance to the source assuming the optical/infrared counterpart is an F8 star, corresponding to the 2MASS source. Typical parameters of an F8 star favour a low absorption $\left(0.2 \times 10^{22} \mathrm{~cm}^{-2}\right)$ at a distance not greater than $1 \mathrm{kpc}$. At this distance the $2-10 \mathrm{keV}$ unabsorbed luminosity is $\sim 4 \times 10^{31} \mathrm{erg} \mathrm{s}^{-1}$ for the $0.2 \mathrm{keV}$ black body. These parameters suggest that IGR J19308+0530 is most probably a neutron star $\mathrm{XRB}$ in quiescence, or a CV. If it is much closer than $1 \mathrm{kpc}$, and although an F8 companion may be a quite extreme case, the source would rather be a CV.

\subsection{IGR J19378-0617}

A first mention of an INTEGRAL source at this position is given in Molkov et al. (2004). They suggested that the source of the hard X-ray emission was SS 442 (1H 1934-063). The authors, however, suspected the presence of a new source given the $5.6^{\prime}$ offset of their position of the IBIS source and that of SS 442. IGR J19378-0617 was then reported by Bird et al. (2007) at a position slightly different than that of Molkov et al. (2004). It was detected at a $18-60 \mathrm{keV}$ SNR of $5.7 \sigma$. It was promptly suspected to be a Sey 1 AGN. Within the Swift/XRT error box lies an extended 2MASS source. 2MASX J19373299-0613046 is at $1.0^{\prime \prime}$ from the best Swift position. Note that this source is also associated with SS 422/1H 1934-063 mentioned in the catalogue of Molkov et al. (2004). It is a Seyfert 1.5 galaxy at $z=0.011$. The 2MASS source is also reported in several catalogues, while it is clearly detected in the UVWI-filter in the UVOT data. It is a known radio source (NVSS J193733-061304) and a known X-ray source (1RXS J193732.8-061305).

We extracted an average spectrum from the two pointings. The spectrum has $4222 \mathrm{cts}$ for a total exposure of $2910 \mathrm{~s}$ which may indicate that pile up is not negligible. To avoid pile up effect we extracted the source spectrum from an annular region centred on the best source position excluding the 10 central pixels. The outer radius of the annulus was set to 40 pixels. The resultant spectrum has $1201 \mathrm{cts}$. An absorbed power law fits the spectrum 
well $\left(\chi_{v}^{2}=1.1\right.$ for 45 d.o.f.). The value of $N_{\mathrm{H}}$ is compatible with the average value of the absorption along the line of sight, which indicates that IGR J19378-0617 is a Sey 1.5 which is not intrinsically absorbed.

\subsection{IGR J23254+5842}

IGR J23254+5842 was first reported in the third edition of the IBIS catalogue (Bird et al. 2007). It was detected in both the 20-40 keV and 40-100 keV energy ranges, and the 18-60 significance of its detection was $6.3 \sigma$ out of a total of $1780 \mathrm{ks}$ of observations. Within the Swift/XRT error box lies a single 2MASS source. 2MASS J23522211+5845307 is $0.92^{\prime \prime}$ away from the centre of the Swift error box, and it is also bright in the DSS I \& II images. Although a very faint source may be present in the U-filter of the UVOT data, we cannot precisely determine its magnitude.

We extracted a spectrum from the single Swift/XRT pointing. The source is very weak with a 70 cts spectrum for a total of $5902 \mathrm{~s}$ exposure. A simple power law fits the spectrum well $(\mathrm{C}$-statistic value $=19.5$ for 13 bins $)$. Adding an absorbing component improves the statistic to 16.3 for 14 bins. Note that when all parameters are left free to vary they are poorly constrained. We therefore froze the power law photon index to 2 . The prefered value of $N_{\mathrm{H}}$ may indicate that the absorption is intrinsic to the source, but the poor quality of the data prevents us any firm conclusion on that matter.

\section{Discussion and conclusions}

We analysed Swift/XRT observations of 12 IGRs that previously lacked X-ray position at several arcsec accuracy. This lack of fine positions at X-ray energy either prevented a confirmation of the supposed type of the object or simply prevented nature of the object to be found. The refinement of the X-ray positions allowed us to identify potential counterparts at infrared, optical and UV wavelengths for all of them. We also report the detection of six serendipitous sources of unknown nature although in the case of SWIFT J023405.1+322707, a K0 star is the likely counterpart and thus suggests the source has a galactic origin. All IGRs that were formerly suspected to be AGN were confirmed through our analysis as indeed being so. This shows that although the error box of INTEGRAL can contain several candidate counterparts, when an AGN is found inside it is usually also at the origin of the hard X-ray emission. This is especially true for sources that have high galactic latitude $\left(>10^{\circ}\right)$. We confirm that IC $4518 \mathrm{~A}$ is the counterpart to IGR J14579-4308, and therefore that this source is a Sey 2. We also truly identify IGR J19378-0617 as a Sey 1.5 galaxy, with known infrared, radio and X-ray counterparts. In IGR J14579-4308 we detected a soft excess in the X-ray spectrum. Soft excesses have been detected in a large number of X-ray spectra of AGNs (e.g. Porquet et al. 2004). The estimated luminosity of this soft excess is $7.9 \times 10^{40} \mathrm{erg} \mathrm{s}^{-1}$, which is compatible with an origin intrinsic to the AGN. When fitting with a diskpn model instead of a black body, we obtain a lower limit on the inner radius of the disk $R_{\text {in }}>6 R_{\mathrm{G}}$.

For the other sources, we either found in the optical and infrared surveys, faint sources within the Swift error box. In two cases these counterparts may be extended or a blend of sources, which prevents an identification to be given. In two cases $U$ and UVW2 counterparts were found. For IGR J09523-6231 we first proposed a tentative Sey 2 identification. The AGN nature of the source has recently been confirmed. It is, however, a Sey 1.5 (Masetti et al. 2008). For IGR J10147-6354 the identification as an AGN, possibly a Sey 2, seems more secure. We, however, stress that only through optical spectroscopy of the counterpart shall the identification be firmly given. For the others, the fact that these objects have point sources as optical/infrared counterparts may suggest that they are Galactic sources, although this is not a definite proof. Only in some specific cases this is, however, strongly supported by some additional facts. IGR J19308+0530 has an F8 star as the most likely counterpart. It spectrum is indicative of little intrinsic absorption (which may also suggest that it is a close object), and is very soft. IGR J18490-0000 has a $K$-band counterpart. Its spectrum is intrinsically absorbed and resembles that of an XRB. Its position in the direction of the Sagittarius Arm tangent would strengthen its Galactic nature, as the arms of the Galaxy are sites with high-density of sources. Note that these sources lie at Galactic latitudes $<7^{\circ}$ which may suggest that they are associated with the Galactic Plane, further supporting a Galactic origin. In all those cases (but IGR J19308+0530), the power law photon index returned by the spectral fit may suggest they are XRBs, although a more definite identification would require optical spectroscopy of the counterpart, and study of the temporal variability of the X-ray source.

Acknowledgements. J.R. thanks A. Maury for very useful suggestions on early versions of this paper. The authors thank Emrah Kalemci for communications of preliminary results on VV 780 prior to publication. We also warmly thank N. Masetti and I. Bikmaev for pointing us more precise results concerning some of the sources, and the anonymous referee for a very useful report which helped us to improve the paper. We acknowledge the use of data collected with the Swift observatory. This research has made use of the SIMBAD database, operated at CDS, Strasbourg, France. It also makes use of data products from the Two Micron All Sky Survey, which is a joint project of the University of Massachusetts and the Infrared Processing and Analysis Center/California Institute of Technology, funded by the National Aeronautics and Space Administration and the National Science Foundation. This research has made use of the NASA/IPAC Extragalactic Database (NED) which is operated by the Jet Propulsion Laboratory, California Institute of Technology, under contract with the National Aeronautics and Space Administration. We acknowledge the use of NVSS, DSS online catalogues.

\section{References}

Bikmaev, I. F., Sunyaev, R. A., Revnivtsev, M. G., \& Burenin, R. A. 2006, Astron. Lett., 32, 221

Bird, A. J., Barlow, E. J., Bassani, L., et al. 2006, ApJ, 636, 765 Bird, A. J., Malizzia, A., Bazzano, A., et al. 2007, ApJS, 170, 175

Bodaghee, A., Courvoisier, T. J.-L., Rodriguez, J., et al. 2007, A\&A, 467, 585

Burenin, R., Mescheryokov, A., Sazonov, S., et al. 2006, ATel, 883

Burrows, D. N., Hill, J. E., Nousek, J. A., et al. 2005, SSRv, 120, 165

Chaty, S., Rahoui, F., Foellmi, C., et al. 2008, A\&A, in press

Gehrels, N., Chincarini, G., Giommi, P., et al. 2004, ApJ, 611, 1005

Hasegawa, T., Wakamatsu, K., Malkan, M., et al. 2000, MNRAS, 316, 326

Kalemci, E., Boggs, S. E., \& Lund, N. 2005, ATel, 410

Krivonos, R, Revnivtsev, M., Lutovinov, A., et al. 2007, A\&A, 475, 775

Lebrun, F., Leray, J. P., Lavocat, P., et al. 2003, A\&A, 411, L141

Lutovinov, A., Rodriguez, J., Revnivtsev, M., \& Shtykovskiy, P. 2005, A\&A, L41

Masetti, N., Malizia, A., Dean, A. J., Bazzano, A., \& Walter, R. 2006a, ATel, 957 Masetti, N., Mason, E., Bassani, L., et al. 2006b, A\&A, 448, 547

Masetti, N., Morelli, L., Cellone, S. A., et al. 2007, ATel 1033

Masetti, N., Mason, E., Morelli, L. et al. 2008, A\&A, 482, 113

Molkov, S. V., Cherepaschuk, A. M., Lutovinov, A. A., et al. 2004, Astron. Lett., 30,534

Porquet, D., Reeves, J. N., O’Brien, P., \& Brinkmann, W. 2004, A\&A, 422, 85

Revnivtsev, M. G., Sazonov, S. Yu., Molkov, S. V., et al. 2006, Astron. Lett., 32 , 145

Rodriguez, J., Bodaghee, A., Kaaret P., et al. 2006, MNRAS, 366, 274

Roming, P. W. A., Kennedy, T. E., Mason, K. O., et al. 2005, SSRv, 120, 95

Sazonov, S., Revnivtsev, M., Krivonos, R., Churazov, E., \& Sunyaev, R. 2007, A\&A, 462, 57

Tomsick, J. A., Chaty, S., Rodriguez, J., et al. 2006, ApJ, 647, 1309

Winkler, C., Courvoisier, T. J.-L., Di Cocco, G., et al. 2003, A\&A, 411, L1 